\title{
Cuando las víctimas de violencia escolar se convierten en agresores: "¿Quién va a defenderme?"
}

\author{
Estefanía Estévez ${ }^{1}$, Teresa I. Jiménez ${ }^{2}$ y David Moreno ${ }^{3}$ \\ ${ }^{1}$ Universidad Miguel Hernández de Elche, ${ }^{2}$ Universidad de Zaragoza, \\ ${ }^{3}$ Universidad Pablo de Olavide de Sevilla (España)
}

\begin{abstract}
Estudios previos han identificado dos subgrupos de víctimas de violencia escolar: víctimas sumisas y víctimas agresivas. Las víctimas sumisas se caracterizan por su retraimiento ante la situación de violencia, mientras que las víctimas agresivas presentan un comportamiento hostil combinado con la situación de victimización. El presente estudio se centra en el segundo subgrupo y tiene como objetivo analizar posibles factores influyentes en el proceso que conduce del estado de victimización a la implicación en conductas agresivas en la escuela. La asunción de partida se fundamenta en la teoría de Emler que sostiene que el sentimiento de indefensión producido por la situación de victimización, junto con la decepción en las figuras adultas de autoridad ante su fracaso en la protección esperada, generan en el adolescente la necesidad de buscar una reputación antisocial y no conformista que le permita defenderse de los ataques venideros. Para poner a prueba estas relaciones, se ha utilizado una muestra compuesta por 1.319 adolescentes de entre 12 y 16 años. Los análisis estadísticos mediante un modelo de ecuaciones estructurales muestran resultados en la línea de la argumentación de Emler. En la discusión se comentan las implicaciones prácticas de estos resultados.
\end{abstract}

Palabras clave: Adolescencia, victimización, violencia escolar, reputación, actitud hacia la autoridad.

When victims of school violence become the aggressors: Who is going to defend me? Previous studies have identified two subgroups of victims of school violence: submissive victims and aggressive victims. Submissive victims are characterized by their withdrawal in the situation of violence, whilst aggressive victims present an hostile behavior combined with the victimization situation. The present study is focused on the second subgroup, and the objective is to analyze potential influential factors in the process that leads from the victimization status to involving in aggressive behaviors at school. The starting premise is based on Emler's theory supporting that the feeling of lack of defense produced by the victimization situation, together with the deception on adult authority figures due to their failure in the expected protection, generate a need in the adolescent to search for an antisocial and non-conformist reputation that allows them to defend themselves from future attacks. To test these relationships, a sample of 1.319 adolescents from 12 to 16 years was used. Statistical analyses by means of a structural equation model show results that are in line with Emler's argument. Practical implication from findings are commented in the discussion section.

Key words: Adolescence, victimization, school violence, reputation, attitude to authority.

Correspondencia: Estefanía Estévez López. Universidad Miguel Hernández de Elche. Departamento de Psicología de la Salud. Av. Universidad, s/n. Edificio Altamira. CP. 03202, Elche-Alicante (España). E-mail: eestevez@umh.es 
En la literatura científica se han identificado dos subgrupos de víctimas de violencia escolar que reflejan, a su vez, dos maneras distintas de reaccionar frente a las agresiones de sus compañeros. La mayoría de víctimas se caracteriza por presentar sumisión y pasividad ante su agresor, es decir, por mostrar retraimiento ante la situación de violencia (Schwartz, Proctor y Chien, 2001). Sin embargo, los estudios han identificado otro tipo de víctimas que muestran una tendencia a presentar un comportamiento hostil combinado con la situación de victimización. Este subgrupo de víctimas ha recibido distintos nombres y se les conoce como bully/víctimas (Boulton y Smith, 1994), víctimas provocativas (Olweus, 2001) o víctimas agresivas (Schwartz et al., 2001). Aunque no existen demasiados datos sobre prevalencia de ambos subgrupos de víctimas, los escasos trabajos al respecto coinciden en señalar que el grupo de víctimas agresivas es inferior en número al de víctimas sumisas. Así, por ejemplo, el estudio de Olweus (2001) indica que el grupo de víctimas agresivas representa aproximadamente al $10-20 \%$ del total de víctimas.

Sin embargo, aunque proporcionalmente las víctimas agresivas representan un grupo más reducido, se encuentran al mismo tiempo en una situación especial de riesgo frente al desarrollo de problemas de desajuste, como han señalado distintos estudios (Estévez, Murgui y Musitu, 2008). En el trabajo llevado a cabo por Estévez, Martínez y Musitu (2006) se observó que las víctimas agresivas obtenían índices más bajos de autoestima que las víctimas sumisas, especialmente en las dimensiones familiar y escolar. Los resultados del estudio llevado a cabo por Unnever (2005) mostraron que las víctimas agresivas informaban de más problemas de autocontrol, así como de más conflictos familiares en comparación con las víctimas sumisas. Estos datos nos sugieren que las víctimas agresivas representan un grupo de gran relevancia para el estudio empírico, lo que contrasta con la escasez de investigaciones sobre las características diferenciales de este subgrupo de víctimas y, más aún, sobre los mecanismos que predicen su ajuste psicosocial, así como el proceso desencadenante del comportamiento hostil de la víctima.

La investigación previa acerca de la relación entre la victimización, la actitud hacia la autoridad institucional y el comportamiento antisocial y delictivo nos ofrece una fundamentación teórica de base para nuestro estudio, y especialmente los trabajos llevados a cabo desde Reino Unido por Emler y Reicher (1987, 1995, 2005). Estos autores argumentan grosso modo que cuando el adolescente es víctima de acoso o maltrato o se percibe en una situación de riesgo y amenaza ante los demás, confía en el deber de protección de las figuras adultas e instituciones de autoridad; sin embargo, los adultos no siempre ofrecen una protección perfecta, lo que puede resultar en la consiguiente decepción del adolescente en estos agentes, así como en la búsqueda de una alternativa informal de protección frente a los ataques de los iguales. La solución inicial, siguiendo la teoría propuesta por Emler, consiste en la búsqueda de una determinada 
reputación fundamentada en una imagen social de persona anticonformista, rebelde y antisocial. Esta reputación lleva implícita la idea de que la persona es fuerte, valiente y está dispuesta a vengarse de modo violento si fuera atacada de nuevo. Finalmente, la estrategia más eficaz para configurar este tipo de reputación es precisamente la implicación en conductas violentas que ejemplifiquen lo que el adolescente quiere transmitir sobre su persona. La violencia sería el medio para conseguir la reputación antisocial anhelada, asumiendo la idea de que los agresores no pueden ser victimizados.

El objetivo del presente estudio pretende poner a prueba la teoría de Emler (2009) en el contexto escolar, teniendo en cuenta la percepción del adolescente de distintas figuras e instituciones de autoridad tanto informal como formal. Los estudios de Emler se han llevado a cabo con población adolescente delincuente, sin embargo, y dada la inexistencia de investigación en el entorno educativo, consideramos que sus supuestos pueden ser un adecuado punto de partida en el estudio del proceso implicado en la relación victimización-agresión en estudiantes de secundaria. Así, el propósito en este trabajo es analizar la relación existente entre la victimización escolar y la conducta agresiva en adolescentes, considerando las siguientes variables influyentes intermedias: la percepción del clima familiar, la actitud hacia el profesorado/escuela, la actitud hacia la policía/leyes, y la reputación antisocial entre los iguales. Planteamos la hipótesis de que la asociación entre victimización y agresión se explica, al menos en parte: (1) por la percepción de la víctima de las figuras e instituciones de autoridad adulta representadas en la familia (padres), la escuela (profesores) y el sistema legal general (policía y leyes), y (2) por la relación existente entre la actitud hacia estas figuras e instituciones de autoridad y la búsqueda de una reputación antisocial.

\section{METODO}

\section{Participantes}

Participaron en el estudio un total de 1.319 adolescentes de ambos sexos (53\% chicas y $47 \%$ chicos) y con edades comprendidas entre los 12 y los 16 años $(M=13.7$; $D T=1.6)$. Los adolescentes estaban escolarizados en siete escuelas de Enseñanza Secundaria Obligatoria de la Comunidad Valenciana (provincias de Alicante y Valencia).

\section{Instrumentos}

Escala de Victimización de Mynard y Joseph (2000). Este instrumento se compone de 20 ítems con una escala tipo Likert de cuatro puntos ( $1=$ nunca, $4=$ muchas veces) que mide tres dimensiones: (1) Victimización verbal (referente a la utilización de insultos, amenazas o humillaciones expresadas verbalmente; ej.: "Algún compañero me ha insultado"), (2) Victimización física (referente a la utilización de agresiones de carácter físico contra la víctima; ej.: "Algún compañero me ha pegado o golpeado") y 
(3) Victimización relacional (referente a la utilización de estrategias cuyo objetivo es el aislamiento social de la víctima; ej.: "Algún compañero le ha dicho a los demás que no se relacionen conmigo"). El alpha de Cronbach de estas tres dimensiones fue $.89, .71 \mathrm{y}$ .70 respectivamente.

Escala de Clima Familiar de Moos, Moos, y Trickett (1984). Utilizamos la subescala Relaciones compuesta por 27 ítems binarios (verdadero-falso) que mide tres dimensiones: (1) Cohesión (grado de compromiso y apoyo familiar percibido por los hijos; ej.: "En mi familia, realmente nos ayudamos y apoyamos los unos a los otros"), (2) Expresividad (grado en que se expresan emociones en el seno familiar; ej.: "En mi familia comentamos nuestros problemas personales"), y (3) Conflicto (grado en que se expresa ira y conflicto entre los miembros familiares; ej.: "En mi familia nos criticamos frecuentemente los unos a los otros"). El alpha de Cronbach de estas dimensiones fue $.86, .73$ y .85 respectivamente.

Escala de Actitud hacia la Autoridad de Emler y Reicher (1995), compuesta por 20 ítems que evalúan la actitud del adolescente hacia las instituciones y figuras de autoridad formal, con un rango de respuesta de 1 (nada de acuerdo) a 4 (totalmente de acuerdo). Esta escala mide dos dimensiones: (1) Actitud hacia la escuela y el profesorado (ej.: "En el colegio no me valoran como persona", "Los profesores tratan mejor a los estudiantes de clases sociales superiores"), y (2) Actitud hacia la policía, las leyes y normas socialmente establecidas (ej.: "La policía está para hacer una sociedad mejor para todos", "Es normal saltarse la ley si no se causa daño a nadie"). El alpha de Cronbach obtenido para estas dimensiones fue .76 y .57 respectivamente.

Escala de Reputación Social de Carroll, Hattie, Durkin, K. y Houghton (1999). Este instrumento se compone de 15 ítems que evalúan la reputación del estudiante entre sus iguales como persona antisocial y no conformista (ej. "Soy un chico/a duro/a", "Me salto las normas"), con una escala de 4 alternativas de respuesta ( 1 = nunca, 4 = siempre). Los ítems miden dos dimensiones: (1) Reputación percibida (“Los demás piensan que yo...") y, (2) Reputación ideal ("Me gustaría que los demás pensasen que yo...”). El alpha de Cronbach obtenida para estas dimensiones fue $.85 \mathrm{y}$ .78 respectivamente.

Escala de Conducta Violenta de Little, Henrich, Jones y Hawley (2003). Esta escala mide, con 24 ítems y un rango de respuesta que oscila entre 1 (muy en desacuerdo) y 4 (muy de acuerdo), comportamientos que implican agresiones hacia otros. El instrumento mide tres dimensiones: (1) Violencia pura (conductas violentas que implican violencia física o verbal; ej.: "Soy una persona que pega a los demás"), (2) Violencia reactiva (conductas violentas como respuesta a la percepción de una agresión previa; ej.: "Cuando alguien me enfada, le hago daño o le hiero") y (3) Violencia instrumental (conductas violentas como medio para conseguir un fin; ej.: "Amenazo a 
otros para conseguir lo que quiero"). El alpha de Cronbach de las tres dimensiones fue $.79, .82$ y .84 respectivamente.

\section{Procedimiento}

Los centros colaboradores en el estudio se seleccionaron aleatoriamente de entre el total de escuelas públicas de Enseñanza Secundaria Obligatoria de la Comunidad Valenciana. Tras acordar con la dirección de los centros educativos su participación voluntaria, se realizó un seminario informativo con la plantilla de profesorado para explicar los objetivos, importancia y alcance del estudio. A continuación se envió una carta explicativa de la investigación a los padres y madres de los alumnos mediante correo postal donde, además, se solicitó que expresaran por escrito su consentimiento en relación con la participación de su hijo. Se acordaron fechas para realizar el pase de cuestionarios con los alumnos, que tuvo lugar en sus aulas habituales, durante un período regular de clase de aproximadamente 45 minutos de duración y previa explicación de los aspectos más relevantes del estudio. En todos los casos, la participación de los adolescentes fue voluntaria y anónima.

\section{RESULTADOS}

En primer lugar se calcularon las correlaciones de Pearson entre todas las variables objeto de estudio, como análisis preliminar de las relaciones existentes entre las dimensiones de los instrumentos seleccionados. Estos datos se presentan en la tabla 1. Puesto que se observaron correlaciones significativas entre variables en el sentido esperado, todas ellas fueron incluidas en los análisis posteriores.

Tabla 1. Correlaciones de Pearson entre las dimensiones objeto de estudio y significación estadística

\begin{tabular}{|c|c|c|c|c|c|c|c|c|c|c|c|c|c|}
\hline Variables & 1 & 2 & 3 & 4 & 5 & 6 & 7 & 8 & 9 & 10 & 11 & 12 & 13 \\
\hline 1. Victimización verbal & 1 & & & & & & & & & & & & \\
\hline 2. Victimización física & $.603(* *)$ & 1 & & & & & & & & & & & \\
\hline 3. Victimización relacional & $.794(* *)$ & $.582(* *)$ & 1 & & & & & & & & & & \\
\hline 4. Cohesión & $-.119(* *)$ & $-.160(* *)$ & $-.164(* *)$ & 1 & & & & & & & & & \\
\hline 5. Expresividad & $-.060(*)$ & -.045 & $-.071(*)$ & $.457(* *)$ & 1 & & & & & & & & \\
\hline 6. Conflicto & $.126(* *)$ & $.129(* *)$ & $.148(* *)$ & $-.565(* *)$ & $-.243(* *)$ & 1 & & & & & & & \\
\hline $\begin{array}{l}\text { 7. Actitud hacia } \\
\text { escuela-profesor }\end{array}$ & $-.085(* *)$ & $-.107(* *)$ & $-.074(*)$ & $.272(* *)$ & $.171(* *)$ & $-.183(* *)$ & 1 & & & & & & \\
\hline $\begin{array}{l}\text { 8. Actitud hacia leyes- } \\
\text { policía }\end{array}$ & -.039 & $-.066(*)$ & $-.058(*)$ & $.211(* *)$ & $.117(* *)$ & $-.143(* *)$ & $.511(* *)$ & 1 & & & & & \\
\hline 9. Reputación percibida & $.082(* *)$ & $.093(* *)$ & $.083(* *)$ & $-.227(* *)$ & $-.141(* *)$ & $.228(* *)$ & $-.410(* *)$ & $-.410(* *)$ & 1 & & & & \\
\hline 10. Reputación ideal & .011 & $.075(*)$ & .037 & $-.164(* *)$ & $-.081(* *)$ & $.148(* *)$ & $-.290(* *)$ & $-.332(* *)$ & $.678(* *)$ & 1 & & & \\
\hline 11. Violencia pura & $.238(* *)$ & $.217(* *)$ & $.241(* *)$ & $-.242(* *)$ & $-.109(* *)$ & $.237(* *)$ & $-.352(* *)$ & $-.278(* *)$ & $.454(* *)$ & $.312(* *)$ & 1 & & \\
\hline 12. Violencia reactiva & $.126(* *)$ & $.151(* *)$ & $.143(* *)$ & $-.253(* *)$ & $-.114(* *)$ & $.208(* *)$ & $-.355(* *)$ & $-.300(* *)$ & $.411(* *)$ & $.318(* *)$ & $.602(* *)$ & 1 & \\
\hline 13. Violencia instrumental & $.170(* *)$ & $.206(* *)$ & $.224(* *)$ & $-.220(* *)$ & $-.112(* *)$ & $.207(* *)$ & $-.343(* *)$ & $-.283(* *)$ & $.389(* *)$ & $.280(* *)$ & $.685(* *)$ & $.591(* *)$ & 1 \\
\hline
\end{tabular}

A continuación, mediante el programa EQS 6.0 (Bentler, 1995) se calculó un modelo de ecuaciones estructurales para profundizar en la comprensión de la interacción entre las variables del estudio. En particular, el modelo se estimó con el objeto de analizar la asociación entre la victimización y la conducta violenta en la escuela, 
considerando como variables influyentes intermedias: la percepción del clima familiar, la actitud hacia el profesorado y la escuela, la actitud hacia la policía y las leyes, y la reputación antisocial entre los iguales. El modelo se compone, por tanto, de 6 factores. Cada factor se calculó a partir de varios indicadores o variables observables; estos indicadores se corresponden con las dimensiones medidas en las escalas utilizadas para la recogida de datos. Así, los factores latentes denominados Clima Familiar, Victimización y Conducta Violenta se componen por 3 indicadores cada uno. El factor latente Reputación se compone de 2 indicadores. Finalmente, los factores latentes Actitud hacia la escuela/profesores y Actitud hacia la policía/leyes, están formados por un solo indicador y, por tanto, presentan una saturación factorial de 1 y un error igual a 0 . Los factores relativos a la percepción de la familia, la escuela y el sistema legal general se calcularon de manera que puntuaciones elevadas indican percepciones negativas.

\begin{tabular}{lc}
\multicolumn{1}{l}{ Tabla 2. Saturaciones factoriales, errores estándar y probabilidad asociada } \\
\hline Variables & $\begin{array}{c}\text { Cargas } \\
\text { factoriales }\end{array}$ \\
\hline Victimización & $1^{\mathrm{a}}$ \\
\hline Victimización verbal & $0.663^{* *}$ \\
Victimización física & $(0.031)$ \\
& $0.958^{* *}$ \\
Victimización relacional & $(0.034)$ \\
\hline Clima familiar & \\
\hline Cohesión & $0.677^{* *}$ \\
& $(0.51)$ \\
Expresividad & $0.531^{* *}$ \\
& $(0.46)$ \\
Conflicto & $1^{\mathrm{a}}$ \\
\hline Actitud escuela/profesores & $1^{\mathrm{a}}$ \\
\hline Actitud policía/leyes & $1^{\mathrm{a}}$ \\
\hline Reputación & \\
\hline Reputación percibida & $0.716^{* *}$ \\
Reputación ideal & $(0.041)$ \\
$1^{\mathrm{a}}$
\end{tabular}

a Fijados en 1.00 durante la estimación. $* * p<.01$

Para determinar la bondad de ajuste del modelo y la significación estadística de los coeficientes, se utilizaron estimadores robustos debido a la desviación de la normalidad de los datos (coeficiente Mardia normalizado: 6.38). El modelo calculado ajustó bien a los datos, como indican los siguientes índices: $C F I=.97, I F I=.97$, $N N F I=.96$, y RMSEA $=.047$. Para los índices CFI, IFI y NNFI se consideran aceptables valores superiores a .95, y para el índice RMSEA valores inferiores a 05 (Batista y Coenders, 2000). Este modelo explica el $35 \%$ de la varianza de la variable final, 
Conducta Violenta. La figura 1 muestra la representación gráfica del modelo estructural final con los coeficientes estandarizados y su probabilidad asociada.

Figura 1. Modelo estructural final con coeficientes de relación y significación estadística

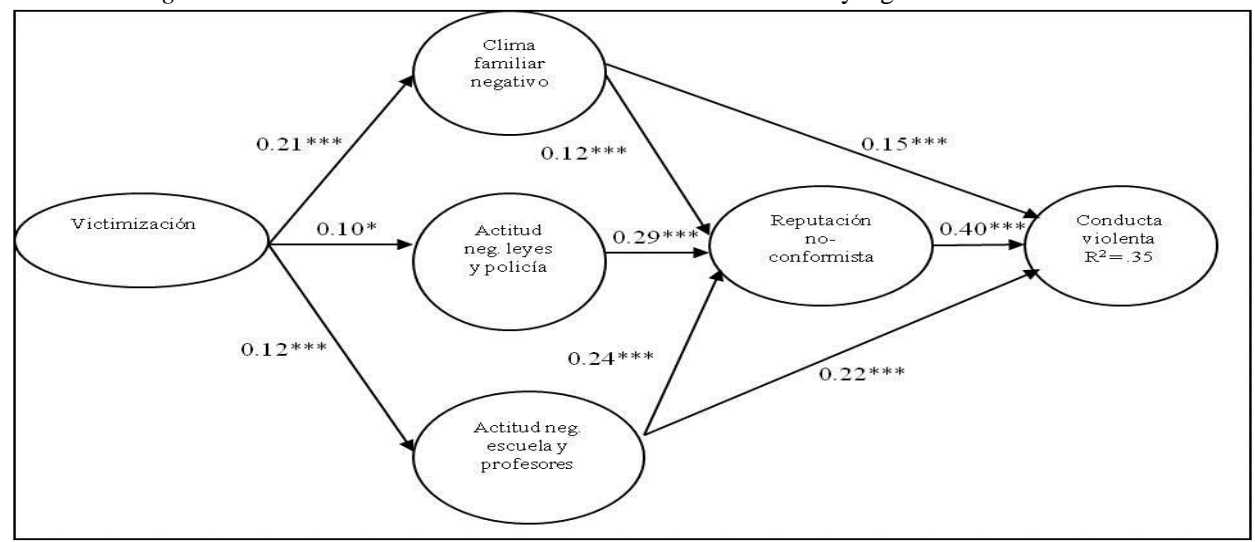

${ }^{*} p<.05 ; * * p<.01 ; * * * p<0.001$

Los resultados del modelo nos muestran una relación indirecta entre la victimización y la conducta violenta. Así, por un lado, observamos relaciones significativas entre el hecho de ser victimizado y la percepción del adolescente de un clima familiar negativo $(\beta=.21, p<.001)$, la actitud negativa hacia la policía y las leyes $(\beta=.10, p<.05)$, y la actitud negativa hacia la escuela y el profesorado $(\beta=.12, p<.001)$. A su vez, estos tres factores mostraron una relación directa y positiva con la reputación no conformista del adolescente entre sus iguales $(\beta=.12, p<.001 ; \beta=.29, p<.001 ; \mathrm{y}$ $\beta=.24, p<.001$ respectivamente). Es decir, los resultados indican que la percepción negativa de las figuras e instituciones de autoridad como la familia, la escuela y la policía, se asocia positivamente con la intención del adolescente de configurar y mantener una reputación antisocial y no conformista entre sus iguales. Finalmente, observamos una estrecha relación positiva entre la reputación y la conducta violenta en la escuela $(\beta=.40 ; p<.001)$. Asimismo, el modelo también muestra la existencia de relaciones directas entre la percepción del clima familiar y la actitud hacia la escuela y el profesorado con la implicación en conductas violentas $(\beta=.15, p<.001$; y $\beta=.22$, $p<.001$ respectivamente).

\section{DISCUSION}

El presente estudio tenía como objetivo analizar el rol desempeñado por determinadas variables en la explicación del proceso que puede desencadenar que una 
víctima de agresiones escolares se convierta también en agresor hacia sus iguales, es decir, en el caso particular de la denominada víctima agresiva. A este respecto, y siguiendo la teoría propuesta por Emler (2009), en nuestra hipótesis de partida manifestamos que se esperaba encontrar relaciones influyentes intermedias con la percepción del adolescente de las figuras e instituciones de autoridad más relevantes en el período adolescente, como son la familia (padres), la escuela (y profesorado) y la policía (y las leyes y normas sociales que representa). Nuestros resultados van en la línea de las conclusiones extraídas por Emler a partir de sus estudios con adolescentes delincuentes en el Reino Unido, y que en el caso del presente trabajo se han visto confirmadas con una muestra de estudiantes escolarizados en nuestro contexto nacional y en relación con comportamientos de victimización y agresión entre iguales.

En particular, nuestros resultados señalan la importancia de la evaluación que realiza el adolescente victimizado de sus contextos sociales más inmediatos y en los que se adscriben las figuras de autoridad más relevantes y que se encuentran en situación de deber ante el desempeño de un papel protector y de proposición de soluciones a la situación de victimización, como son la familia, la escuela y el sistema legal general. Así, las relaciones observadas en el modelo nos indican que cuando la víctima no confía en el apoyo y consuelo que puede recibir por parte de padres, profesores y otras figuras de autoridad como la policía, es más probable que opte por una vía alternativa de autoprotección a través de la configuración de una reputación antisocial y no conformista frente a los iguales que eventualmente derive en un comportamiento agresivo que refuerce dicha imagen social.

La investigación previa ha destacado la estrecha asociación existente entre el hecho de ser o haber sido victimizado y el comportamiento delictivo de la víctima (Shafer y Ruback, 2002; Wiebush, Freitag y Baird, 2001), sin embargo, estos estudios se han llevado a cabo con población delincuente y fuera de nuestras fronteras, principalmente en Reino Unido y Estados Unidos. La investigación en nuestro país se encuentra en sus inicios, máxime si atendemos a la población menor escolarizada y que presenta ambos problemas conjuntamente -victimización y conducta agresiva- y que se encuentra, por tanto, en una situación de desajuste psicosocial de alto riesgo. Por tanto, consideramos como aspecto fundamental que la investigación futura siga ahondando en el análisis de la situación específica de las víctimas agresivas, tanto en sus características individuales como en las particularidades de los entornos inmediatos que rodean al adolescente en esta situación, puesto que como nuestros resultados señalan, una clave importante se encuentra en la percepción que tienen las víctimas de las figuras adultas de autoridad y protección.

Las implicaciones prácticas de estos resultados son de suma relevancia. Niños y adolescentes obedecen las normas socialmente establecidas porque confían en los modelos adultos que las sustentan y esperan, a cambio de esta obediencia y confianza, 
que los adultos aboguen por un trato de respeto y respondan con el apoyo y protección requeridos ante determinadas situaciones, por el hecho de representar agentes de autoridad y poder. Trabajar en esta línea parece fundamental para que los jóvenes que sufren situaciones de victimización en la escuela no se sientan defraudados, desamparados y desprotegidos ante esos abusos. El clima familiar positivo en base a una comunicación empática y afectiva entre padres e hijos ha sido identificado en la literatura científica como uno de los principales factores de protección frente al desarrollo de conductas agresivas en la adolescencia (Estévez, Murgui, Musitu y Moreno, 2008; Stevens, De Bourdeaudhuij y Van Oost, 2002), por lo que el trabajo en esta línea sigue siendo fundamental. De igual modo, la percepción de un clima escolar positivo donde el adolescente valora la relación con sus compañeros y profesores, de los que percibe atención y apoyo, se ha vinculado con la presencia de menos problemas de agresión en la escuela (Blankemeyer, Flannery y Vazsonyi, 2002). Por último, también la colaboración conjunta entre la familia y la escuela merece más atención. La formación de padres y madres en colaboración con las escuelas, así como el desarrollo de cursos y talleres específicos para el profesorado acerca de las señales que permiten una detección precoz de situaciones de victimización, o los recursos necesarios para afrontar situaciones de violencia en el aula, pueden desencadenar repuestas más ajustadas para víctimas y agresores y, principalmente, prevenir situaciones de victimización.

\section{Agradecimientos}

Este estudio ha sido elaborado en el marco del proyecto de investigación PSI2008-01535/PSIC: "Violencia escolar, victimización y reputación social en la adolescencia" subvencionado por el Ministerio de Ciencia e Innovación de España.

\section{REFERENCIAS}

Batista, J.M. y Coenders, G. (2000). Modelos de ecuaciones estructurales. Madrid: La Muralla.

Bentler, P.M. (1995). EQS structural equations program manual. Encino, CA: Multivariate Software.

Blankemeyer, M., Flannery, D.J. y Vazsonyi, A.T. (2002). The role of aggression and social competence in children's perceptions of the child-teacher relationship. Psychology in the Schools, 39(3), 293-304.

Boulton, M.J. y Smith, P.K. (1994). Bully/victim problems in middle-school children: Stability, self-perceived competence, peer perceptions, and peer acceptance. British Journal of Developmental Psychology, 12, 315-329.

Carroll, A., Hattie, J., Durkin, K. y Houghton, S. (1999). Adolescent reputation enhancement: Differentiating delinquent, nondelinquent, and at-risk youths. Journal of Child Psychology and Psychiatry, 40, 593-606.

Emler, N. (2009). Delinquents as a minority group: Accidental tourists in forbidden territory or voluntary émigrés? In F. Butera y J. Levine (Eds.). Coping with minority status: Responses to exclusion and inclusion. US: Cambridge University Press. 
Emler, N. y Reicher, S. (1987). Orientations to institutional authority in adolescence. Journal of Moral Education, 16, 108-116.

Emler, N. y Reicher, S. (1995). Adolescence and delinquency. Oxford, UK: Blackwell Publishers Ltd.

Emler, N. y Reicher, S. (2005). Delinquency: Cause or consequence of social exclusion? En D. Abrams, M.A. Hogg y J.M. Marques (Eds.), The Social Psychology of Inclusion and Exclusion (pp. 211-241). New York: Psychology Press.

Estévez, E., Martínez, B. y Musitu, G. (2006). La autoestima en adolescentes agresores y víctimas en la escuela: La perspectiva multidimensional. Intervención Psicosocial, 12, 32-41.

Estévez, E., Murgui, S. y Musitu, G. (2008). Psychosocial adjustment in aggressors, pure victims and aggressive victims at school. European Journal of Education and Psychology, 1(2), 29-39.

Estévez, E., Murgui, S., Musitu, G. y Moreno, D. (2008). Adolescent aggression: effects of gender and family and school environments. Journal of Adolescence, 31, 433-450.

Little, T.D., Henrich, C.C., Jones, S.M. y Hawley, P.H. (2003). Disentangling the "whys" from the "whats" of aggressive behaviour. International Journal of Behavioral Development, 27, 122-131.

Moos, R.M., Moos, B.S. y Trickett, E.J. (1984). FES, WES y CES Escalas de Clima Social. Madrid: TEA Ediciones.

Mynard, H. y Joseph, S. (2000). Development of the multidimensional Peer Victimization Scale. Aggressive Behavior, 26, 169-178.

Olweus, D. (2001). Peer harassment: A critical analysis and some important issues. En J. Juvonen y S. Graham (Eds), Peer harassment in school: The plight of the vulnerable and victimized (pp. 3-20). New York: Guilford Press.

Shaffer, J.N. y Ruback, R.B. (2002). Violent victimization as a rick factor for violent offending among juveniles. OJJDP Juvenile Justice Bulleting. Washington, D.C.: U.S. Department of Justice, Office of Juvenile Justice and Delinquency Prevention.

Schwartz, D., Proctor, L.J. y Chien, D.H. (2001). The aggressive victim of bullying: Emotional and behavioral dysregulation as a pathway to victimization by peers. En J. Juvonen y S. Graham (Eds), Peer harassment in school: The plight of the vulnerable and victimized (pp. 147-174). New York: Guilford Press.

Stevens, V., De Bourdeaudhuij, I. y Van Oost, P. (2002). Relationship of the family environment to children's involvement in bully/victim problems at school. Journal of Youth and Adolescence, 31, 419-428.

Unnever, J.D. (2005). Bullies, aggressive victims, and victims: Are they distinct groups? Aggressive Behavior, 31, 153-171.

Wiebush, R., Freitag, R. y Baird, C. (2001). Preventing delinquency through improved child protective services. OJJDP Juvenile Justice Bulleting. Washington, D.C.: U.S. Department of Justice, Office of Juvenile Justice and Delinquency Prevention.

Recibido: 2 de diciembre de 2009

Recepción Modificaciones: 19 de enero de 2010

Aceptado: 22 de enero de 2010 